ARTICLE

\title{
Perception of the COVID-19 pandemic among people with spinal cord injury: an Italian survey
}

\author{
Gabriele Righi $\mathbb{D}^{1 凶}$, Emanuele Baroni ${ }^{2}$, Lorenzo Righi ${ }^{3}$, Laura Belloni ${ }^{2}$ and Giulio Del Popolo ${ }^{1}$ \\ (C) The Author(s), under exclusive licence to International Spinal Cord Society 2022
}

STUDY DESIGN: An observational study based on an online survey to explore if the participant had experienced (1) cancellation or delay of scheduled health services (2) reduction of assistance provided by a caregiver (3) barriers to social participation and recreational activities. Three validated questionnaires to investigate well-being and symptoms of anxiety and depression were also administered.

OBJECTIVES: Our main aim was to quantify the obstacles experienced by adults living with SCI in Italy during COVID-19 pandemic, to explore the presence of depression and anxiety symptoms and to quantify subjective well-being.

SETTING: Outpatient clinic of a Spinal Unit in Italy.

METHODS: Online survey via direct contact and by e-mail lists.

RESULTS: In total, 101 individuals completed the survey. Of, 82.2\% participants reported a history of deferment or cancellation of non-COVID-19 health services. The majority (56.4\%) revealed that, at least seldom, they have chosen to reduce their usual everyday activities and more than one third (37.6\%) affirmed that they had been forced to renounce to one or more of their occupations. Discontinuation of assistance by caregivers was uncommon. The median score of questionnaires measuring depression and anxiety symptoms do not differ significatively when compared with prior studies. The variable that explored the limitations experienced in everyday activities showed a significant correlation with the results of the questionnaires measuring well-being and symptoms of anxiety.

CONCLUSIONS: We believe that our results could contribute to the discussion ongoing inside our community on how to answer to the new challenges of this pandemic period and of the post-pandemic future.

Spinal Cord Series and Cases (2022)8:2 ; https://doi.org/10.1038/s41394-021-00470-x

\section{INTRODUCTION}

COronaVIrus Disease2019 (COVID-19) pandemic is putting a strain on various features of the lives of people affected by spinal cord injury $(\mathrm{SCl})$.

Healthcare organizations often reduced the services offered, as regards particularly outpatient visits, outpatient rehabilitation and non-COVID-19 diagnostic services, with a risk of late detection and treatment of complications such as urological pathologies or pressure sores, despite telemedicine services tried to maintain patients in adequate follow up. The relationships between adults living with $\mathrm{SCl}$ and their caregivers were endangered by the apprehension of contagion by SARS-CoV-2, given the impossibility of maintaining an adequate interpersonal distance while assisting with personal care. In addition, health authorities and governments have enforced lockdown measures to limit the spread of this disease, restricting the possibility of traveling, closing nonessential businesses, forbidding recreational and amateur sports activities. These nonpharmaceutical interventions have shown effectiveness in reducing COVID-19 incidence and mortality [1] but they also may yield adverse consequences, such as a potential increase in stress, social isolation and symptoms of anxiety and depression.
A large survey conducted in Italy during April 2020, around 4 weeks into strict lockdown measures, showed a high incidence of depression symptoms and anxiety symptoms between the general population [2].

There are very few published data regarding the overall impact of COVID-19 pandemic on mental health of people affected SCI and, to our best knowledge, no study has been conducted on this topic in Italy, a nation that holds one of the highest COVID-19 burdens worldwide. We therefore distributed an online survey to individuals with $\mathrm{SCl}$, investigating their mental health and the eventual occurrence, during the last year, of issues with their caregivers or limitations of access to healthcare services and to their usual everyday activities.

Our main aim was to quantify the obstacles experienced by adults living with $\mathrm{SCl}$ in Italy during COVID-19 pandemic, to explore the presence of depression and anxiety symptoms and to quantify subjective well-being; the secondary goal was to investigate the eventual correlation of mental health and wellbeing with the limitations experienced in their daily activities and the difficulties encountered in accessing healthcare services or in their relationship with caregivers.

\footnotetext{
${ }^{1}$ Spinal Unit, Careggi University Hospital, Florence, Italy. ${ }^{2}$ Regional Center for Critical Relationships, Careggi University Hospital, Florence, Italy. ${ }^{3}$ Clinical Trial Center, Careggi
} University Hospital, Florence, Italy. ${ }^{\circledR}$ email: righiga@aou-careggi.toscana.it 


\section{METHODS}

\section{Survey development}

The authors reviewed existing surveys and manuscripts regarding the impact of COVID-19 pandemic on mental health [2-6] and then interviewed experts in the field of spinal cord medicine. Upon that foundation, we have drafted an online survey composed by 5 parts. Each part of the survey was displayed on a single web page and had to be completed in order to progress to the next page. In the first part we addressed participants' demographic and injury-specific data (sex, age, neurological level of injury and AIS grade, as determined according to ASIA/ISCoS International Standards for Neurological Classification of Spinal Cord Injury) and asked whether they had contracted COVID-19 or not. In case of an affirmative answer to this latter, there was a further question, investigating if they had been hospitalized or not. The second part of the survey was made of seven different items, all of which were 5-point Likert scales, with possible answers ranging from "almost always" to "never". Items 1 to 3 investigated if the participant had experienced cancellation or delay of scheduled outpatient visits, outpatient rehabilitation programs or non-COVID-19 diagnostic respectively. Questions 4 and 5 inquired about eventual reduction of assistance provided by a caregiver, based on a decision of the participant (item 4) or of the caregiver itself (item 5). Item 6 questioned if the participant had suffered a reduction of his/her everyday activities for a lack of assistance, while item 7 asked if the participant had reduced his/her everyday activities in fear of contagion by COVID-19. Part 3,4 and 5 were three validated questionnaires, General Anxiety Disorder-7 (GAD-7) [7] Patient Health Questionnaire-9 (PHQ-9) [8] and the 5-item World Health Organization Well-Being Index (WHO-5) [9] respectively. We used the Italian version of all the three questionnaires. No identifying details were collected. Only adults (aged $\geq 18$ years) were allowed to participate. Consent was explicitly provided by every participant before accessing the survey, in accordance with the General Data Protection Regulation of the European Union.

\section{Survey distribution}

The survey was distributed to people living with $\mathrm{SCl}$ in two ways: via direct contact with outpatients of the Spinal Unit of Florence and by e-mail lists maintained by various associations, foundations and support groups affiliated to the Federazione Associazioni Italiane Paratetraplegici (FAIP, federation of Italian associations of para-tetraplegics) The survey had been accessible from March 1st 2021 to April 30th 2021, for a total of 61 days.

\section{Statistical analysis}

In the descriptive analysis, median and percentages are reported. 3 multivariate linear regression models were elaborated to investigate the eventual presence of correlations between GAD-7, PHQ-9, WHO-5 and the items composing part 2 of the survey. For the purpose of analysis, part 2 items were aggregated in three categories: everyday activities, access to healthcare and assistance by caregiver. Results were adjusted by age, sex, level of lesion (paraplegic vs tetraplegic) AIS grade (motor complete, A and $B$, vs motor incomplete, C and D) history of COVID-19 infection. Statistical tests were two-tailed. Level of significance was set at $p=0.05$. Analyses were carried out using R v4.0.0 (https://www.r-project.org)

\section{RESULTS}

A total of 101 individuals from 13 different regional areas of Italy (out of a total of 20) completed the survey. 37 were enrolled via direct contact of outpatients while 64 participated after receiving the web-link to the survey by e-mail (potential respondents 626, response rate $10.22 \%$ ) Subjects' median age was 53 years (interquartile range: 47-61) the majority of participants were male ( $66.3 \%$ male vs $33.7 \%$ female) and there was a slight prevalence of paraplegic (56.4\%) and motor complete lesions $(53,4 \%) 8$ individuals reported to have contracted COVID-19 and 4 of them required hospitalization, with a complete recovery after hospital discharge. Participants that experienced at least some cancellation or postponement (with answers ranging from 2, seldom, to 5, almost always) of outpatient visits were $47,5 \%$ of the total. The percentage of people that reported a history of deferment or cancellation of non-COVID-19 diagnostic services was 31.7\%, while it was $37.6 \%$ as regards outpatient rehabilitation. A total of $82.2 \%$ participants had encountered difficulties in obtaining at least one of the three types of aforementioned healthcare treatment, i.e. gave a score comprised between 2 and 5 to at least one of the three questions. The data regarding barriers in accessing healthcare services are summarized in Table 1. As regards caregivers, only a small minority $(13.9 \%$, considering the total of answers from 2 to 5) of the participants revealed that they had asked their caregivers to reduce their services; an even lower percentage, $12.9 \%$, faced a refusal from their caregivers to guarantee the usual level of assistance. On the other hand, the majority $(56.4 \%)$ of participants reported that, at least seldom, they have chosen to reduce their usual everyday activities due to the fear of contagion and more than one third (37.6\%) affirmed that they had been forced to renounce to one or more of their occupations due to lack of assistance. The median score of GAD-7 was 5. Utilizing, as suggested in the original paper [7] cut points of 5,10 , and 15 as representing mild, moderate, and severe levels of anxiety, the percentage of participants above the first, second and third cut point were $45.5 \%, 22.8 \%$, and $9.9 \% .25 .7 \%, 11.9 \%$, and $9.9 \%$ of people reported mild, moderate and severe depressive symptoms, with a PHQ-9 total median score of 5 . As regards wellbeing, median score of WHO-5 was 72 (within a 0-100 range). We found no significant association between the score of GAD-7, PHQ-9 and WHO-5 and access to healthcare and assistance by caregiver. The variable that explored the limitations experienced in everyday activities showed a significant correlation with the results of the three tests: GAD-7 ( $t$ value 3.070, $p<0,01)$, PHQ-9 $(t$ value $2.501, p<0.05)$ and WHO-5 ( $t$ value $-2.850, p<0.01)$.

\section{DISCUSSION}

In this paper, we tried to investigate all the main ways in which COVID-19 pandemic is having an impact on people affected by $\mathrm{SCl}$.

It was clear since the beginning of the pandemic [10] that there was a high risk of deprioritization for access to healthcare and support services for people living with $\mathrm{SCl}$ and that the pandemic would have put a strain on mental health of both general population $[2,3]$ and people affected by disability [11].

Considering the COVID-19 pandemic, the World Health Organization (WHO) itself stated that additional considerations from governments, healthcare systems and disability service providers are needed for people with disabilities and their support network, since a global pandemic has the potential to significantly increase the daily challenges of people with disabilities and may have a greater impact compared to the general population [12].

One of the most interesting data of our survey, in our opinion, is that a very high percentage of participants, $82.2 \%$, reported that they had suffered cancellation or postponement of outpatient

Table 1. Total number of participants divided upon their answer to questions regarding if they had experienced a delay or cancellation of outpatient visits, non-COVID-19 diagnostics or outpatient rehabilitation.

\begin{tabular}{|c|c|c|c|c|c|}
\hline & Never & Seldom & Sometimes & Often & Almost always \\
\hline Outpatient visits & 53 & 18 & 14 & 7 & 9 \\
\hline Non-COVID-19 diagnostics & 69 & 12 & 6 & 9 & 5 \\
\hline Outpatient rehabilitation & 63 & 7 & 14 & 7 & 10 \\
\hline
\end{tabular}


visits, non-COVID-19 diagnostics or outpatient rehabilitation. This very high percentage highlights all the limitations and difficulties of Italian territorial services, but is coherent with the available data on the reduction of rehabilitation services in Europe during the first phases of the pandemic -March to May 2020- when outpatient services have been completely stopped in $83 \%$ of the European countries, including $87 \%$ of the population [13].

A highly discussed topic in literature about COVID-19 and disability is the role of caregivers, given the clear incompatibility of assisting a person with disability and maintaining an adequate interpersonal distance [14] but in the overwhelming majority of the population that answered to our survey the relationship with their caregivers was never interrupted, even for a brief time: the main reason for that, in our opinion, is that in Italy there is a strong prevalence of familial caregivers.

A recent publication by García-Rudolph et al. [15] demonstrated that depression was significantly higher than before COVID-19 and social integration significantly decreased. They interviewed adults with chronic $\mathrm{SCl}$ and compared the score of three questionnaires with data obtained before the beginning of COVID-19 pandemic.

These results are partially supported by our findings, with a relevant percentage of participants that complained about a reduction of their usual daily activities, due to both fear of contagion and lack of assistance.

On the other hand, the median score of GAD-7 and PHQ-9 was quite low in our sample, and slightly less than $10 \%$ had a score indicative for severe depression or severe anxiety.

The percentage of self-reported "caseness" of anxiety were similar to the results of a vast meta-analysis published in 2016 [16].

As regards symptoms of depression, both the median score of PHQ-9 and the percentage of persons above the cut-off for severe depression were similar to the findings of two large studies by Bombardier et al. [17] and by Richardson et al. [18].

The lower intensity of symptoms of anxiety and depression in the participants in our survey when compared to the Italian general population [2] may be surprising, but it is reasonably due to the different time frame of the pandemic when the two surveys were taken: while the other one was performed four weeks into the initial strict lockdown, our survey had been online from March 1st 2021 to April 30th 2021, when, even if several limitations to gatherings and recreational activities were still in act, the vaccination campaign was already ongoing.

Finally, it is worth of consideration that, although more people lamented difficulties in accessing healthcare services than in maintaining their usual level of activities, only the score of the items assessing the latter significantly correlated with GAD-7, PHQ-9 and WHO-5. A possible explanation is that visits, exams or rehabilitation activities that were postponed in the first phases of the pandemic could have been already rescheduled at the time of answering the survey. It is to be considered also that a reasonable explanation for this correlation may be the reduced social participation of people affected by anxiety or depression. We think that, in any case, this finding should support wider accessibility to social participation and recreational activities for persons affected by SCl.

\section{Limitations}

This study has several important limitations. First, our distribution method introduced a partial bias. The help provided by associations and support groups in the recruitment of participants implied that most of the responders had a link with them and therefore may be protected against social isolation and loneliness.

Further, we did not know if the omitted examinations and therapies had negative consequences on participants' health or not.

Another limitation is that, since our survey was anonymous, we were not able to make any comparison with previous measurements of mental health and well-being.
When investigating the effects of COVID-19 pandemic on healthcare accessibility, relationships with caregivers and social participation we considered the pandemic period as a whole and therefore we could have overlapped pictures related to different time frames.

\section{CONCLUSIONS}

Although limited, this is one of the few studies to investigate the impact of COVID-19 pandemic on various features which are very relevant for people affected by $\mathrm{SCl}$ : access to healthcare, assistance by caregivers, social participation, mental health, wellbeing. We believe that our results could contribute to the discussion ongoing inside our community on how to answer to the new challenges of this pandemic period and of the (hopefully forthcoming) post-pandemic future.

In our opinion, the most important message that we can deliver is that guaranteeing accessibility to social participation and recreational activities for persons affected by $\mathrm{SCl}$ should be a considered, using a word that have strongly characterized our last several months, essential.

\section{DATA AVAILABILITY}

The datasets generated during the current study are available from the corresponding author on reasonable request.

\section{REFERENCES}

1. Haug N, Geyrhofer L, Londei A, Dervic E, Desvars-Larrive A, Loreto V, et al. Ranking the effectiveness of worldwide COVID-19 government interventions. Nat Hum Behav. 2020;4:1303-12

2. Rossi R, Socci V, Talevi D, Mensi S, Niolu C, Pacitti F, et al. COVID-19 pandemic and lockdown measures impact on mental health among the general population in Italy. Front Psychiatry. 2020;11:790. Aug 7.

3. Pfefferbaum B, North CS. Mental health and the Covid-19 pandemic. N Engl J Med. 2020;383.6:510-2

4. Cao W, Fang Z, Hou G, Han M, Xu X, Dong J, et al. The psychological impact of the COVID-19 epidemic on college students in China. Psychiatry Res. 2020;287:112934.

5. Rossi R, Socci V, Pacitti F, Di Lorenzo G, Di Marco A, Siracusano A, et al. Mental health outcomes among frontline and second-line health care workers during the coronavirus disease 2019 (COVID-19) pandemic in Italy. JAMA Netw open. 2020;3: e2010185-e2010185.

6. Greenberg N. Mental health of health-care workers in the COVID-19 era. Nat Rev Nephrol. 2020;16:425-6.

7. Spitzer RL, Kroenke K, Williams JB, Löwe B. A brief measure for assessing generalized anxiety disorder: the GAD-7. Arch Intern Med. 2006;166:1092-7.

8. Spitzer RL, Kroenke K, Williams JB.Validation and utility of a self-report version of PRIME-MD: the PHQ primary care study. Patient Health Questionnaire Primary Care Study Group, \& Patient Health Questionnaire Primary Care Study Group. JAMA. 1999;282:1737-44.

9. Staehr JK. The use of well-being measures in primary health care-the DepCare project. World Health Organization, Regional Office for Europe: Well-Being Measures in Primary Health Care-the DepCare Project. Geneva: World Health Organization, 1998

10. O'Connell CM, Eriks-Hoogland I, Middleton JW. Now, more than ever, our community is needed: spinal cord injury care during a global pandemic. Spinal Cord Ser Cases. 2020;6:18.

11. Turk MA, McDermott S. The COVID-19 pandemic and people with disability. Disabil Health J. 2020;13:100944.

12. World Health Organization. (2020). Disability considerations during the COVID-19 outbreak (No. WHO/2019-nCoV/Disability/2020.1). World Health Organization.

13. Negrini S, Grabljevec K, Boldrini P, Kiekens C, Moslavac S, Zampolini M. et al. Up to 2.2 million people experiencing disability suffer collateral damage each day of COVID-19 lockdown in Europe. Eur J Phys Rehabil Med. 2020;56:361-5.

14. Sánchez-Raya J, Sampol J. Spinal cord injury and COVID-19: some thoughts after the first wave. Spinal Cord. 2020;58:841-3.

15. García-Rudolph A, Saurí J, López Carballo J, Cegarra B, Wright MA, Opisso E.et al. The impact of COVID-19 on community integration, quality of life, depression and anxiety in people with chronic spinal cord injury. J Spinal Cord Med. 2021:1-10.

16. Le J, Dorstyn D. Anxiety prevalence following spinal cord injury: a meta-analysis. Spinal Cord. 2016;54:570-8. 
4

17. Bombardier CH, Richards JS, Krause JS, Tulsky D, Tate DG. Symptoms of major depression in people with spinal cord injury: implications for screening. Arch Phys Med Rehabil. 2004;85:1749-56.

18. Richardson EJ, Richards JS. Factor structure of the PHQ-9 screen for depression across time since injury among persons with spinal cord injury. Rehabil Psychol. 2008;53:243.

\section{COMPETING INTERESTS}

The authors declare no competing interests.

\section{ADDITIONAL INFORMATION}

Correspondence and requests for materials should be addressed to Gabriele Righi.

Reprints and permission information is available at http://www.nature.com/ reprints

Publisher's note Springer Nature remains neutral with regard to jurisdictional claims in published maps and institutional affiliations. 